\title{
Modeling the Evolution of Line-of-Sight Blockage for V2V Channels
}

\author{
Mate Boban, Xitao Gong, and Wen Xu \\ Huawei Technologies Duesseldorf $\mathrm{GmbH}$, European Research Center \\ Riesstr. 25c, 80992, Munich, Germany \\ Email: \{mate.boban, xitao.gong, wen.dr.xu\}@huawei.com
}

\begin{abstract}
We investigate the evolution of line of sight (LOS) blockage over both time and space for vehicle-to-vehicle (V2V) channels. Using realistic vehicular mobility and building and foliage locations from maps, we first perform LOS blockage analysis to extract LOS probabilities in real cities and on highways for varying vehicular densities. Next, to model the time evolution of LOS blockage for V2V links, we employ a three-state discrete-time Markov chain comprised of the following states: i) LOS; ii) non-LOS due to static objects (e.g., buildings, trees, etc.); and iii) non-LOS due to mobile objects (vehicles). We obtain state transition probabilities based on the evolution of LOS blockage. Finally, we perform curve fitting and obtain a set of distance-dependent equations for both LOS and transition probabilities. These equations can be used to henerate time-evolved $\mathrm{V} 2 \mathrm{~V}$ channel realizations for representative urban and highway environments. Our results can be used to perform highly efficient and accurate simulations without the need to employ complex geometry-based models for link evolution.
\end{abstract}

\section{INTRODUCTION}

In contrast to existing cellular networks that focus primarily on increasing the mobile data rates, fifth-generation $(5 \mathrm{G})$ network is expected to efficiently support the so-called vertical industries (e.g., industrial, eHealth, and automotive vertical, among other). Supporting automotive vertical, in the form of vehicle-to-anything (V2X) communication, is seen as one of the most challenging tasks for $5 \mathrm{G}$ networks, particularly in terms of end-to-end latency and reliability [1].

One of the key distinguishing features of V2X communication is the high mobility, possibly on both sides of the link (as in the case of $\mathrm{V} 2 \mathrm{~V}$ communication). Another salient aspect of $\mathrm{V} 2 \mathrm{X}$ communication is that it is often related to safety, either directly (e.g., emergency braking, intersection collision avoidance application [2], etc.) or indirectly (e.g., platooning, lane-change maneuvers, etc.). To ensure that V2X communication systems can support the application requirements efficiently, a key initial step is realistically defining the channel characteristics for different environments (e.g., urban, highway, rural) and V2X communication types (e.g., V2V, V2I, V2P). Given the V2X application requirements, one of the most relevant aspects of channel modeling is the time evolution of $\mathrm{V} 2 \mathrm{~V}$ links and the related concept of spatial consistency. Time and space evolution of LOS blockage refers to time-consistent realization of LOS blockage for $\mathrm{V} 2 \mathrm{~V}$ channels, based on the location of the transmitter $(\mathrm{Tx})$, the receiver $(\mathrm{Rx})$, and the composition of their surroundings. Consistent LOS blockage realization is important in order to assign the appropriate path loss, shadowing, small-scale, and large-scale parameters over time and space. This implies that there should be a continuity in terms of vehicle locations over time (i.e., requiring a continuous vehicle movement) and in terms of scatterer distribution around the vehicles.

In the realm of the 3GPP channel modeling (e.g., SCME model [3]), which resort to independent "drops" of devices in space and time, time evolution and spatial consistency have not been taken into consideration. On the other hand, time evolution and spatial consistency are inherently supported by geometry-based deterministic models (e.g., [4], [5]). However, for performing efficient simulations it is also beneficial to have a model that can generate consistent channel realizations for a chosen environment without the need for complex geometric modeling using location-specific map information. While V2X channel measurements and modeling have attracted considerable attention in recent years [6], a comprehensive geometry-based stochastic model for time and space evolution of LOS blockage is currently not available for $\mathrm{V} 2 \mathrm{~V}$ channels.

In this paper, we attempt to fill this gap by performing a comprehensive study of time evolution of $\mathrm{V} 2 \mathrm{~V}$ links in urban and highway environments. We employ a Markov chain comprised of three states: i) LOS; ii) NLOSb - non-LOS due to static objects (e.g., buildings, trees, etc.); and iii) NLOSv non-LOS due to mobile objects (vehicles), since these three states were shown to have distinct path loss and shadowing parameters [5], [7]. To calculate LOS and transition probability statistic ${ }^{1}$, we perform geometry-based deterministic simulations of LOS blockage and extract the parameters from real cities and highways for LOS blockage and transition probabilities. Based on the empirical results from five large cities (downtown Rome, New York, Munich, Tokyo, and London) and a highway section (10 km, including two- and three-lane per direction as well as on-ramp traffic), we perform curve fitting and obtain a set of distance-dependent polynomial equations for both LOS and transition probabilities. To test whether the parameters extracted from a set of cities can generalize to other cities, we compare the model parameterized on the five cities to downtown Paris. The results show a high correlation for both LOS probabilities and transition probabilities. Our results can be used to generate time- and space-consistent LOS blocking for V2V channels by assigning appropriate parameters for each of the three states in representative urban and highway environments.

The most important contributions of this work are as follows.

- We perform a systematic large-scale analysis of LOS blockage and transition probability using real locations of vehicles and other objects to arrive at a practical model for time evolution of V2V links in real urban and highway environments;

- We incorporate the moving environment (vehicles) into the calculations of LOS and transition probability and show that its impact is significant in both urban and highway environment; we also show that, due to the low height of both Tx and Rx antennas, vehicle density has a strong impact on LOS and transition probabilities, thus requiring separate LOS blockage analysis for low, medium, and high density of vehicular traffic.

- We extract parameters that can be used to perform efficient simulations of time-evolved V2V channels, without the need for complex geometry-based deterministic modeling.

\footnotetext{
${ }^{1}$ Throughout the paper, we use the term "LOS probabilities" to encompass the probability of LOS, NLOSb, and NLOSv.
} 
The rest of the paper is organized as follows. Section II discusses existing work on spatially consistent V2V channels. Section III describes the model for time evolution of V2V links and the tools we use for estimating the LOS and transition probabilities. Section IV] shows the LOS and transition probability parameterization results, whereas Section V discusses model validation, usage, and comparison with a state of the art model. Section VI concludes the paper.

\section{RELATED WORK}

While geometry-based deterministic models intrinsically support time evolution and spatial consistency, stochastic models, (either geometry-based or not) need additional mechanisms to support these features. While LOS probability has been extensively explored in the literature for cellular systems (for example, see [8]), the first steps to achieve time- and space-consistent models have been discussed only recently in the community [9]. For V2X and other dual-mobility communication systems such as device-to-device (D2D) communication, this is an even more challenging problem, since mobility on both sides of the link makes the modeling more complicated.

Markov chains are often used to efficiently model the timedependent evolution of different systems. They have been used to characterize different aspects of wireless channels. GilbertElliot burst noise channel, a two state hidden Markov model, has been used to model the bit error probabilities for a wireless channel [10], [11]. Similarly, modeling time evolution of V2V links using Markov chains has been previously explored in literature. Dhoutaut et al. [12] propose a shadowing-pattern model for V2V links that uses two-state Markov chains to determine the level of shadowing caused by other vehicles on an urban street [12]. Similarly, Abbas et al. [7] use the same model to quantify the shadowing effect of obstructing vehicles on V2V links in car following scenario in highway and urban environments. Both studies employ a small set of measurements to validate the model. Wang et al. [13] extend the Markov model to include V2V link cross-correlation (correlation of two geographically close V2V links).

However, while the above papers employ Markov chains to model time evolution of $\mathrm{V} 2 \mathrm{~V}$, they are based on limited measurement or simulation data applicable to a single scenario. To the best of our knowledge, our work is the first to perform a largescale study to realistically parameterize probability of being in each of the three states (LOS, NLOSv, NLOSb) as well as the transition probabilities in both urban and highway environments.

The whitepaper by key 3GPP stakeholders [9] noted that the missing components in 3GPP standardized channel models, both above and below $6 \mathrm{GHz}$, include: i) spatially consistent LOS probability/existence; ii) blockage modeling; and iii) moving environment (e.g., cars). This paper provides a model that contributes to the solution of these shortcomings for $\mathrm{V} 2 \mathrm{~V}$ communication.

\section{Model for Time Evolution of V2V Channels}

There exist measurement studies that analyze the LOS blockage of $\mathrm{V} 2 \mathrm{~V}$ links using video recordings collected during the measurements (e.g., [14], [15], [16]). However, extracting a general model for LOS blockage evolution using the limited amount of data collected therein is not possible, since the data does not encompass the blockage behavior across an entire environment (be it city or highway) or for different traffic conditions. Additionally, such studies may be imprecise, because they rely on estimation of LOS blockage inferred from videos.

In this work, we resort to a large-scale simulation study, which enables us to analyze V2V links between large number of vehicles

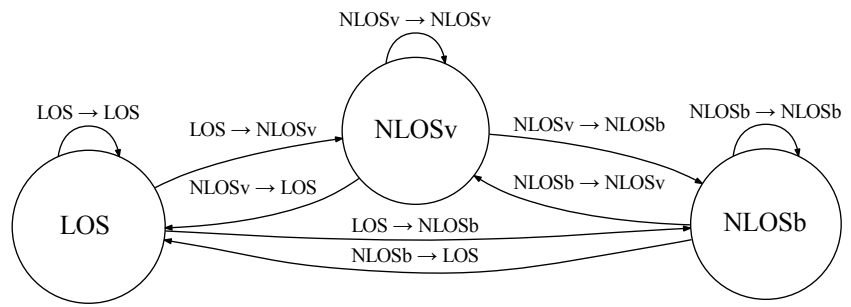

Figure 1. Markov chain for modeling the time evolution of V2V links. States: Line-of-Sight (LOS); ii) non-LOS due to static objects (NLOSb); and iii) non-LOS due to mobile objects (NLOSv).

in various environments and with different vehicle densities. To model the time evolution of $\mathrm{V} 2 \mathrm{~V}$ links, we apply a three-state discrete-time Markov chain, as shown in Fig. 1. This is in contrast to state of art where two states (LOS and NLOS) are usually assumed for $\mathrm{V} 2 \mathrm{~V}$ channels. The probability of the states and the transition probabilities are parameterized through extensive simulation.

\section{A. Mobility modeling}

In order to obtain realistic V2V LOS blockage behavior from simulations, vehicles need to move in a realistic way over real roads. For this reason, we used SUMO (Simulation of Urban MObility) [17] to generate vehicular mobility in cities and on highway. By using real roadways and traffic rules and employing vehicular traffic dynamics models such as car-following [18], SUMO is capable of generating accurate vehicle positions, speeds, inter-vehicle distance, acceleration, overtaking and lane-changing maneuvers, etc. Previous work (e.g., [5], [12]) has shown that $\mathrm{V} 2 \mathrm{~V}$ channel characteristics are affected by the density of vehicular traffic. To that end, for each environment, we generated three traffic densities, qualitatively characterized as low, medium and high. The densities in urban areas were generated according to values proposed by Ferreira et al. [19], whereas for highways we use traffic flow measurement values reported by Wisitpongphan et al. [20]. To allow the vehicular traffic to move into steady state, we run the mobility model for 300 seconds in each environment before performing the LOS blockage analysis. We used one second as time step for mobility simulation.

\section{B. Datasets used for training the model}

1) Urban: To determine LOS and transition probabilities in urban environments, we extracted the roadway (used for mobility simulation) and object outlines (buildings, trees, walls, etc.) from OpenStreetMap [21] for downtown areas of five cities: downtown Rome, New York, Munich, Tokyo, and London. The reason for using multiple cities is to obtain a set of "generic" urban parameters, i.e., those that can readily describe a typical urban environment in terms of LOS blockage, mobility patterns, road configurations, etc. The criteria for selecting the cities were practical: we selected those cities where the object outlines available in OpenStreetMap are as complete as possible. Additionally, we analyzed whether the vehicular mobility simulation generated by SUMO contained any anomalies (e.g., complete gridlocks due to incorrect intersection configurations or disconnected road segments). From the initially considered 10 large cities, we selected downtown Rome, New York, Munich, Tokyo, and London (along with Paris, used for testing the model) based on the described criteria. In cities, all simulated vehicles were personal cars. More details on the locations used can be found in Table I.

Note that, while the areas used for modeling are of limited size, for high vehicle density there were up to a hundred thousand $\mathrm{V} 2 \mathrm{~V}$ communication pairs per time step in the urban area since, 
Table I

LOCATIONS AND VEHICLE DENSITIES USED FOR ANALYSIS

Environments

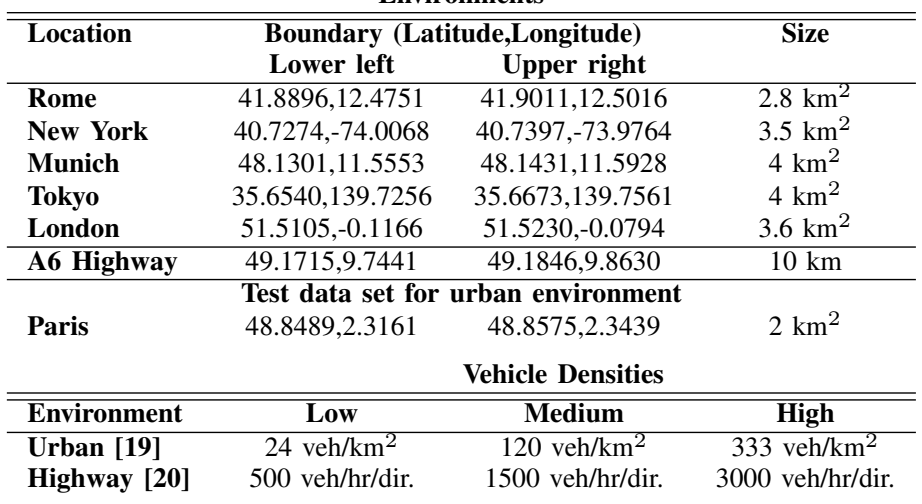

for a given vehicle, all other vehicles within $500 \mathrm{~m}$ radius were considered as possible V2V communication pairs. For medium and high vehicular density, we perform the simulations for 300 seconds. For low density, the number of vehicles and therefore V2V links was smaller, which required 1000-second simulation runs to obtain a representative sample size.

2) Highway: We performed highway simulations with SUMO on a $10 \mathrm{~km}$ stretch of A6 highway between Braunsbach and Wolpertshausen in Bavaria, Germany. The road alternates between two and three lanes per direction and has several entry/exit ramps. We simulated the traffic so that $80 \%$ of the vehicles enter and exit at the each end of the highway, whereas remaining $20 \%$ enter and exit over two ramps near the beginning and the end of the stretch. Since vehicle speeds and dimensions affect the LOS blockage results, $10 \%$ of the simulated vehicles were trucks and remaining were personal cars. Some buildings and forest exist on each side of the highway; they can block LOS for larger Tx-Rx distances, particularly for the vehicles moving on the entry/exit ramps. For medium and high density, we perform the simulations for 1000 seconds. For low density, we perform simulations for 2000 seconds. Note that different highways will have different configurations in terms of lane numbers, surrounding objects, etc, which will affect LOS blockage. It is difficult to encompass all different variations, therefore we focus on the most common highway configuration.

\section{LOS blockage analysis}

We start our analysis by acknowledging that V2V links can have their LOS blocked by two distinct object types, static and mobile, which have distinct impact on V2V links [5]. Furthermore, static objects such as buildings, trees, etc., typically block the LOS for V2V links between vehicles that are on different roads (e.g., perpendicular roads joined by intersections). On the other hand, mobile objects (predominantly other vehicles) block the LOS over the surface of the road.

We use the LOS blockage classification provided by GEMV ${ }^{2}$, a freely available, geometry-based V2X propagation modeling tool [5]. GEMV ${ }^{2}$ uses the outlines of vehicles, buildings, and foliage to distinguish between LOS, NLOSv, and NLOSb links. In order to do so, $\mathrm{GEMV}^{2}$ performs geometry-based deterministic LOS blockage analysis using the outlines of buildings and foliage from OpenStreetMap [21] and vehicular mobility traces from SUMO [17]. Provided that realistic mobility model is used and the OpenStreetMap database contains majority of buildings and foliage, GEMV ${ }^{2}$ produces highly realistic LOS blockage results.

In simulations, we place the antenna in the middle of the roof on vehicles and set its height to $10 \mathrm{~cm}$. For a vehicle to block the V2V link (i.e., for NLOSv blockage to occur), it needs to be within $60 \%$ of first Fresnel zone between the antennas on the communicating vehicles. When the LOS is blocked by both static and mobile objects, we classify this as NLOSb state, because in vast majority of cases static objects such as buildings are the dominant blocking factor. bin.

When determining LOS, GEMV ${ }^{2}$ takes into account the electromagnetic interpretation of LOS, wherein it calculates whether $60 \%$ of first Fresnel ellipsoid is free of any obstructions, in order to determine whether LOS is blocked. That said, we evaluated the LOS blockage results for frequencies between $2 \mathrm{GHz}$ and $6 \mathrm{GHz}$ and the results do not differ significantly. The reason is that the Tx-Rx distances are up to 500 meters, and the resulting difference between frequencies is small; for example, the largest difference between $60 \%$ of first Fresnel zone between $2 \mathrm{GHz}$ and $6 \mathrm{GHz}$ at $500 \mathrm{~m} \mathrm{Tx}-\mathrm{Rx}$ distance is 1 meter. The results showed in the paper refer to $2 \mathrm{GHz}$ carrier frequency.

\section{Transition probability analysis}

In order to obtain the transition probability in the three-state Markov chain shown in Fig. 1, we separate the data based on Tx-Rx distance into 10-meter distance bins and generate the transition probabilities for each of them. For any single step transition between states $a$ and $b$, we have the following transition probability from measurement time step $t$ to $t+1$ and for a given distance bin $d$ :

$$
p_{a b}(d)=\operatorname{Pr}\left(X_{t+1}=b \mid X_{t}(d)=a\right) .
$$

In effect, this creates a set of distance-dependent transition probabilities with one transition probability matrix for each $\mathrm{Tx}$ Rx distance bin. In addition to LOS blockage, GEMV ${ }^{2}$ provides information about transitions between the LOS states. We use the results from $\mathrm{GEMV}^{2}$ to calculate the transition probability using a frequency-based approach: for each LOS state $a$ (where $a \in\{L O S, N L O S v, N L O S b\}$ ), we count the transition from $a$ to state $b$ within a distance bin $d$ (note that $b=a$ is possible, since state $a$ can transition in itself). Then, we divide this number by the total number of transitions from $a$ to all possible states (i.e., total number of occurrences of $a$ ) to obtain the empirical transition probability:

$$
\hat{p}_{a b}(d)=\frac{\sum_{t} \mathbf{1}_{\left(X_{t+1}=b \mid X_{t}(d)=a\right)}}{\sum_{t} \mathbf{1}_{\left(X_{t}(d)=a\right)}} .
$$

where $\mathbf{1}_{A}$ is the indicator of event $A$.

We use the value of time interval equal to one second as a good trade-off between the ability to train the model (shorter time interval would require proportionally more data to obtain representative results) and precision (within one second, in vast majority of cases, there will be at most one transition between LOS states, whereas in a longer period this might not be the case).

\section{MODEL PARAMETERIZATION}

\section{A. LOS blockage}

Fig. 2 shows the LOS probabilities for each of the three states in urban, whereas Fig. 3 shows the results for highway. Results for different vehicle densities show an intuitively expected result: the higher the vehicle density, the more probable the NLOSv state, since in low density scenarios there simply are not that many vehicles around to block the LOS. For high density urban scenario, NLOSv probability reaches $50 \%$ when $\mathrm{Tx}$ and $\mathrm{Rx}$ are between 30 and 70 meters apart. In terms of the relationship between the probabilities of all three states, note that NLOSb remains unaffected by the increased vehicle density. The increase 


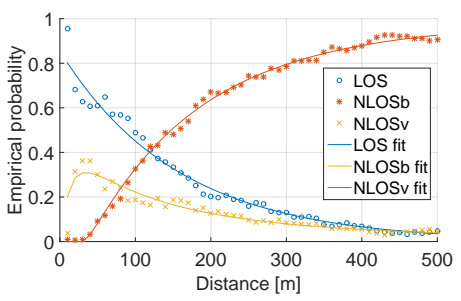

(a) Low Density.

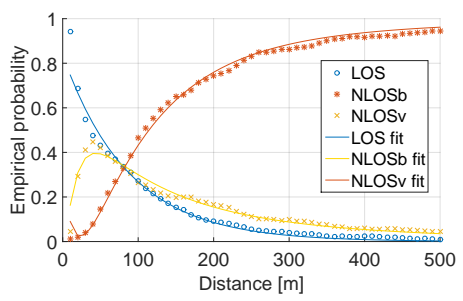

(b) Medium Density.

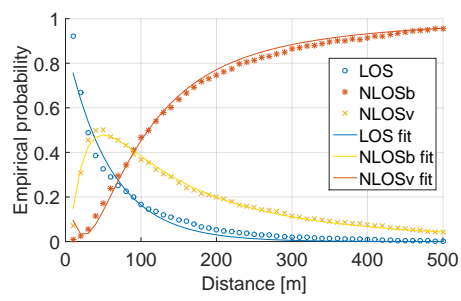

(c) High Density.

Figure 2. LOS probabilities in urban environment: combined Rome, New York, Munich, Tokyo, and London results.

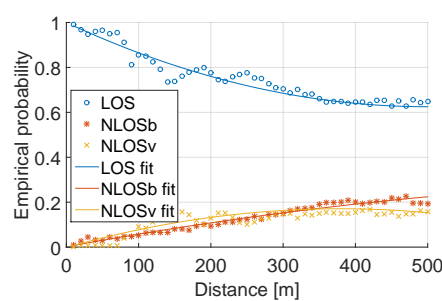

(a) Low Density.

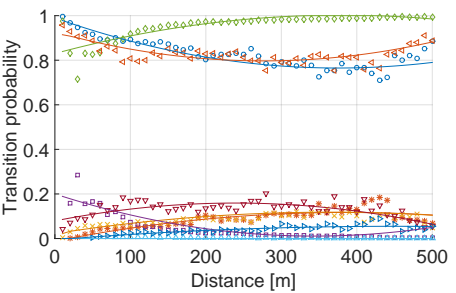

(a) Low Density.

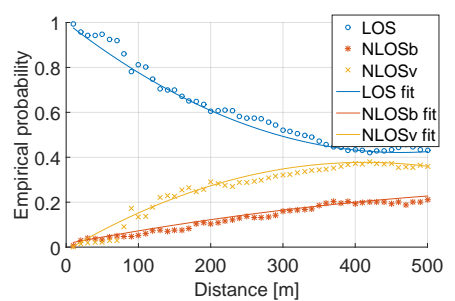

(b) Medium Density.

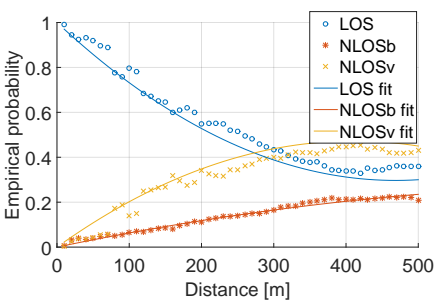

(c) High Density.

Figure 3. LOS probabilities on A6 highway.

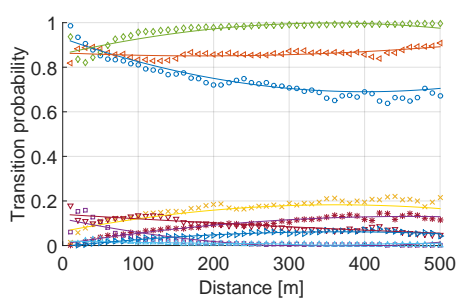

(b) Medium Density.

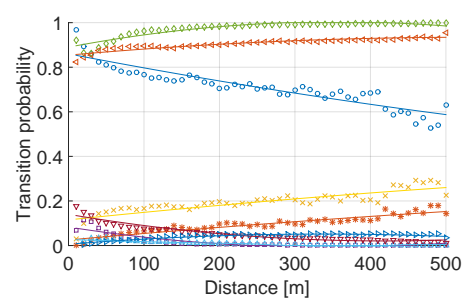

(c) High Density.

Figure 4. Transition probabilities in urban environment: combined Rome, New York, Munich, Tokyo, and London results.

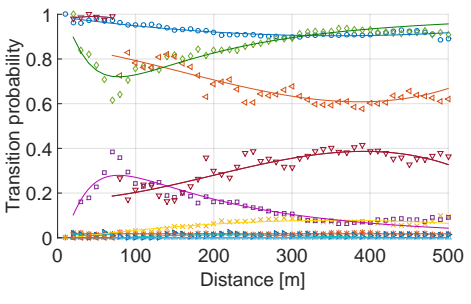

(a) Low Density.

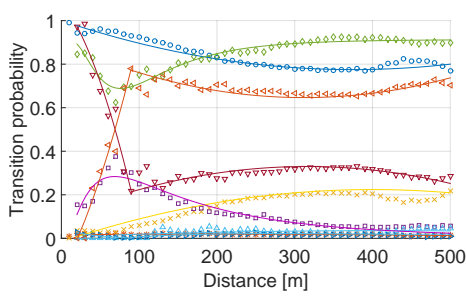

(b) Medium Density.

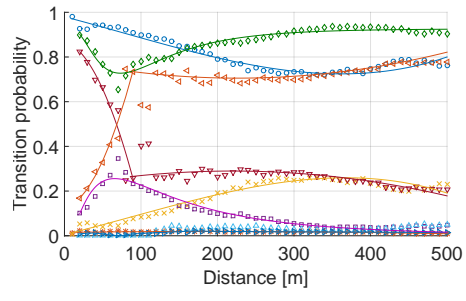

(c) High Density.

Figure 5. Transition probabilities on A6 highway.

in NLOSv probability is at the expense of LOS probability. While the NLOSb in urban is most often caused by buildings lining the roads, in highway scenarios, NLOSb is caused mainly by the forest surrounding the highway, with occasional building creating NLOSb for on- and off-ramp traffic.

\section{B. LOS transition probabilities}

Figs. 4 and 5 shows the state transition probabilities for urban and highway environments, respectively, along with the curve fits according to equations presented in Table III Across densities in urban environment (Fig. 4), LOS transition probability to itself reduces with increasing distance and corresponding transition from LOS to NLOSv and NLOSb increases. The remaining transition probabilities are comparatively independent of distance, with the exception of the expected increase from NLOSb to itself with increasing distance.

Transition probabilities in highway are more dynamic. The most interesting is the relationship between NLOSv-to-NLOSv and NLOSv-to-LOS transitions: with distance increasing from zero to 100 meters, the NLOSv-to-NLOSv rapidly increases, with symmetric decrease in NLOSv-to-LOS transitions. This is a result of car-following behavior of vehicles, where vehicles are following each other at the equivalent of 1-second gap, resulting in 30 to 50-meter distance, thus making NLOSv-to-NLOSv transition increasingly more likely as distance increases. Above 100 meters, 
Table IV

NLOSv-to-NLOSb transitions also come into play, limiting the NLOSv-to-NLOSv increase. Additionally, between 60 and 100 meters, there is a visible spike in NLOSv-to-NLOSv transitions, accompanied by a dip in NLOSv-to-LOS for all vehicle densities. This is again a result of the car-following model. In cases when there are three or more vehicles following each other in the same lane, the middle vehicle is often blocking the LOS between the vehicle in front and behind it. Since the car-following is bound to continue for some time, it results is increased NLOSv probability between front and rear vehicle, which are between 60 and 100 meters apart. Note that the resulting transition probability for low density is somewhat variable because of the smaller number of data points available.

\section{Curve fitting for LOS blockage and transition probabilities}

To provide a tractable model for generation of time-evolved $\mathrm{V} 2 \mathrm{~V}$ links, we perform curve fitting for LOS and transition probabilities. Table III shows the equations of resulting LOS probability curves, whereas Table III shows the equations for transition probabilities.

For LOS probabilities in highway, the equations are in the form of second degree polynomials: $P(L O S)=\boldsymbol{a} d^{2}+\boldsymbol{b} d+\boldsymbol{c}$, where $d$ is the Tx-Rx distance. In urban environment, exponential and log-normal distributions are a better fit for LOS probabilities. Similarly, curve fits for transition probabilities predominantly take the form of second degree polynomials, with the exception of transitions involving NLOSb state in highway environment, which are better approximated by a log-normal distribution. For more complex transition probability curves in highway, we perform piece-wise curve fitting, since there are notable discontinuities, particularly in case of transitions involving NLOSv (e.g., NLOSvto-NLOSv transition: Fig. 5, due to the effect of in-lane carfollowing on LOS blocking by vehicles.

We list LOS probability equations for two out of three curves; the third one can be obtained by subtracting from one the remaining two terms. The same applies for transition probabilities: we show two outgoing probabilities from each state. Note that, due to the imperfections of the curve fitting process, in certain scenarios at low and high distances, the probability equations will result in a probability above one. For this reason, we introduce a ceiling of one for each probability. Similarly, at very low distances (e.g., below 10 meters), the summation of the LOS probability equations (and similarly, summation of outgoing transition probability equations) can amount to more than one. In these situations, we advise to use equation for one of the three probabilities, forcing the lowest of the three probabilities to zero, and subtracting the first probability from one to obtain the third probability.

\section{Validation, Use, and Comparison to State of ART}

\section{A. Validation of the proposed model}

To test how well the proposed model generalizes to other cities (i.e., those on which it was not trained), we compared the model trained on the data from five cities shown in Table I and a data set obtained in downtown Paris (also described in Table I). Table IV shows the results of comparison in terms of Pearson Correlation Coefficient for both the LOS probabilities and transition probabilities. The correlation is above 0.95 for all three LOS probabilities. Similarly, the correlation coefficients for transition probabilities are high (average correlation equals 0.84 ), with only the NLOSv $\rightarrow$ NLOSv below 0.8 . The most likely reason for NLOSv $\rightarrow$ NLOSv discrepancy is that the streets in Paris are significantly narrower and with fewer lanes per direction than in the five cities that the model was trained on. The narrower
CORRELATION COEFFICIENTS: PARIS VS MODEL (TRAINED ON ROME, NEW YORK, MUNICH, TOKYO, AND LONDON DATA SETS). MEDIUM DENSITY.

\begin{tabular}{lccc}
\multicolumn{4}{c}{ LOS Probabilities } \\
\hline \hline Paris/model & LOS & NLOSb & NLOSv \\
Corr. coeff. & 0.9565 & 0.9823 & 0.9528 \\
& & & \\
\multicolumn{4}{c}{ Transition Probabilities } \\
\hline \hline Paris/model & LOS & NLOSb & NLOSv \\
LOS $\rightarrow$ & 0.8037 & 0.9857 & 0.8319 \\
NLOSb $\rightarrow$ & 0.9587 & 0.8316 & 0.8102 \\
NLOSv $\rightarrow$ & 0.8488 & 0.9080 & 0.5851
\end{tabular}

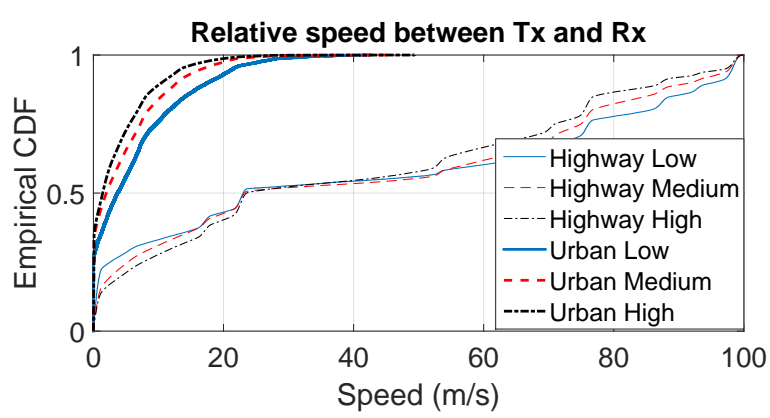

Figure 6. Relative speed between communicating vehicles in highway and urban environments.

street configuration results in higher probability of continued LOS blockage by vehicles. Overall, however, the correlation results show that the model fits well to urban environments outside those that it was trained on and can thus be used with confidence as a representative model for "typical" urban environments.

\section{B. Usage of the model}

Algorithm 1 describes how the developed model can be used to generate LOS states for a V2V link. For $n$ time steps, the algorithm generates a set of $n$-tuple states by using transition equations (Table III). The states represent the time evolution of the link in the given environment and for a given density. Initial state is selected randomly based on the probability of each state given the distance (Figs. 2, 3). Since the model takes as input distance between $\mathrm{Tx}$ and $\mathrm{Rx}$ for each time step, there needs to exist consistency between subsequent distances between Tx and Rx in order to obtain credible results for LOS blockage and transition probabilities. Therefore, we analyze the distribution of speed in each of the six scenarios (highway/urban, low/medium/high density), which combined with the one-second interval results in the $\mathrm{Tx}-\mathrm{Rx}$ distance variation.

Fig. 6 shows the distribution of relative speeds between Tx and $\mathrm{Rx}$; the figure helps in quantifying reasonable distance that the vehicles can travel in a given environment. In urban environments, the relative bearing of two vehicles can be between 0 and 360 degrees, whereas on highway the vehicles travel either in the same or opposite directions, with the slight deviation to this rule caused by on- and off-ramp traffic. Therefore, as shown in Fig. 6, the relative speed in urban areas is mostly limited to 0$20 \mathrm{~m} / \mathrm{s}$, whereas the relative speed on highway will be distinct for same and opposite traffic, with same direction traffic ranging from $0-25 \mathrm{~m} / \mathrm{s}$ and opposite ranging from $50-100 \mathrm{~m} / \mathrm{s}$. Note that the speeds can readily be translated in distance traveled, since the time step under consideration is fixed to one second. For considerably different vehicle speeds (e.g., in case of heavy traffic jams), the LOS blockage and transition probabilities would need to be adjusted accordingly. 
Table II

CURVE FITTING RESULTS: LOS PROBABILITIES

Highway: LOS probability $y$ vs. distance $d: y=\min \left(1, \max \left(0, \boldsymbol{a} d^{2}+\boldsymbol{b} d+\boldsymbol{c}\right)\right)$

\begin{tabular}{lccccccccc}
\hline Density & \multicolumn{3}{c}{ Low } & \multicolumn{4}{c}{ Medium } & \multicolumn{3}{c}{ High } \\
& $\mathbf{a}$ & $\mathbf{b}$ & $\mathbf{c}$ & $\mathbf{a}$ & $\mathbf{b}$ & $\mathbf{c}$ & $\mathbf{a}$ & $\mathbf{b}$ & $\mathbf{c}$ \\
\hline LOS & $1.5 e^{-6}$ & -0.0015 & 1 & $2.7 e^{-6}$ & -0.0025 & 1 & $3.2 e^{-6}$ & -0.003 & 1 \\
NLOSb & $-2.9 e^{-7}$ & 0.00059 & 0.0017 & $-3.7 e^{-7}$ & 0.00061 & 0.015 & $-4.1 e^{-7}$ & 0.00067 & 0
\end{tabular}

Urban: LOS probability $y$ vs. distance $d, d \geq 0: y=\min (1, \max (0, f(d)))$

\begin{tabular}{lccc}
\hline \hline Density & Low & Medium & High \\
\hline LOS & $0.8548 e^{-0.0064 d}$ & $0.8372 e^{-0.0114 d}$ & $0.8962 e^{-0.017 d}$ \\
NLOSv & $\frac{1}{0.0396 d} e^{\frac{\left(-(\ln (d)-5.2718)^{2}\right.}{3.4827}}$ & $\frac{1}{0.0312 d} e^{\frac{\left(-(\ln (d)-5.0063)^{2}\right.}{2.4544}}$ & $\frac{1}{0.0242 d} e^{\frac{\left(-(\ln (d)-5.0115)^{2}\right.}{2.2092}}$
\end{tabular}

Table III

CURVE FITTING RESULTS: TRANSITION PROBABILITIES.

Highway: Transition probability $y$ vs. distance $d: y=\min (1, \max (0, f(d))) \mathbf{d}_{T}=70$ (Low) / 90 (Medium/High)

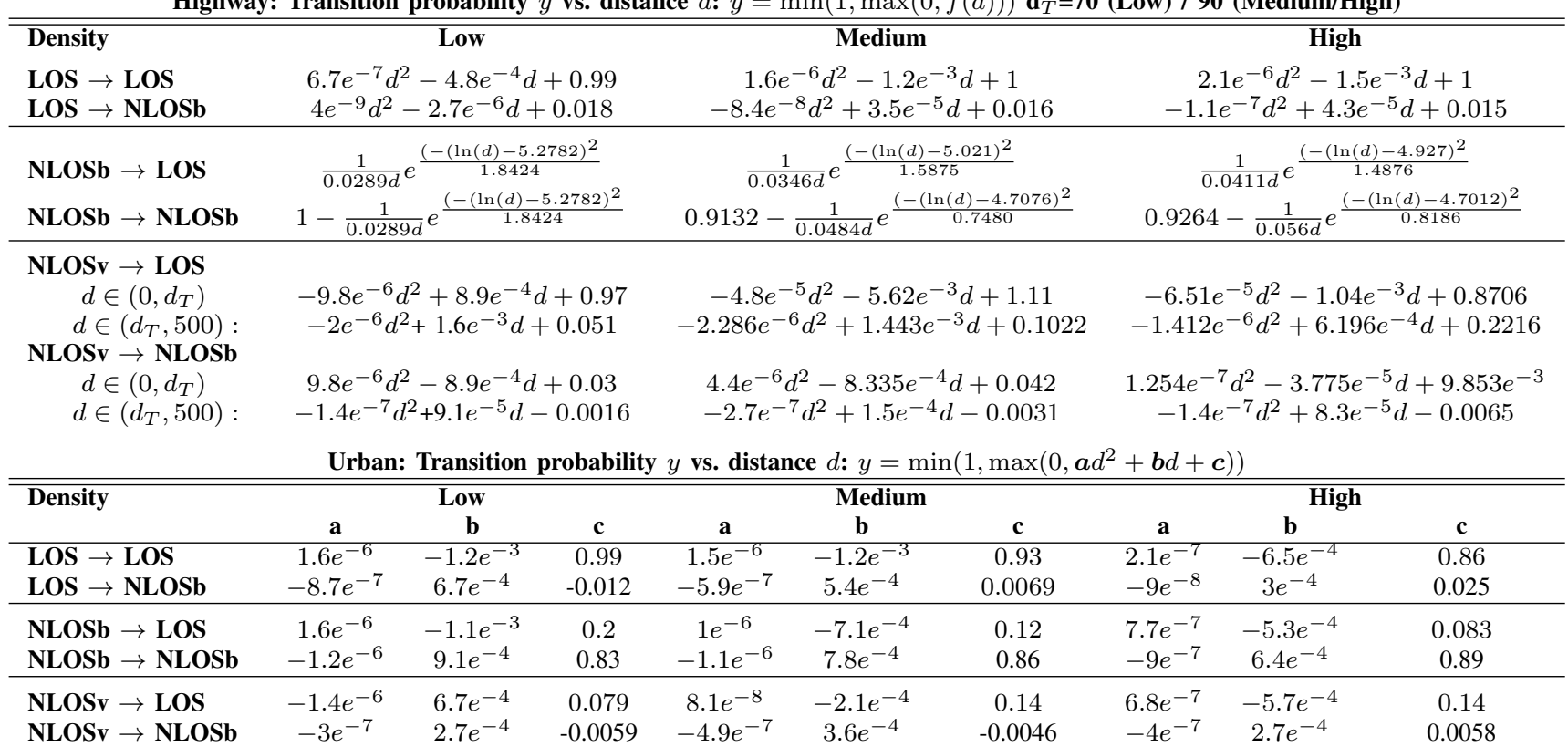

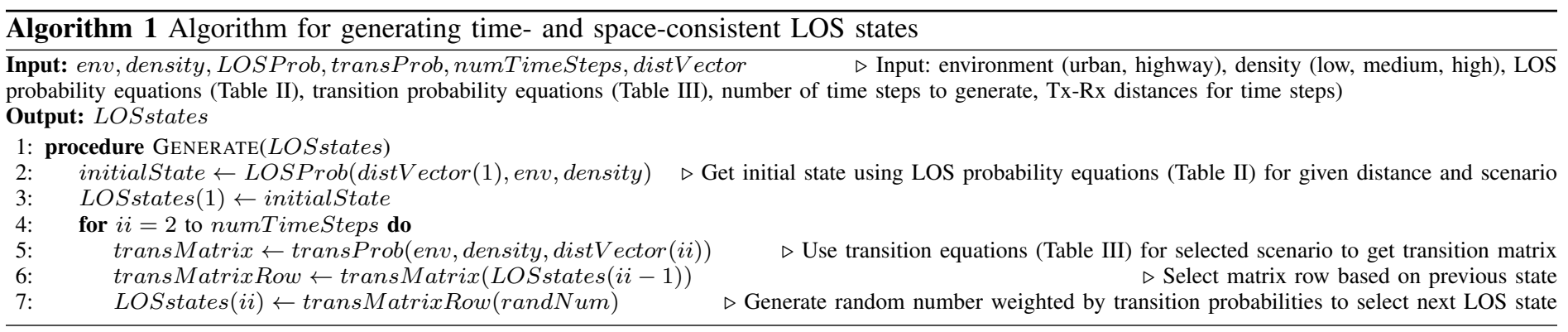

\section{Path loss comparison with non-time-evolved model}

Since there are no comprehensive V2V link LOS blockage and transition probability models available in the literature, we compare the proposed model with the well-established 3GPP/ITU Urban Micro (UMi) LOS probability model [8], which is currently also used for D2D communication:

$$
P(L O S)=\min \left(\frac{d_{1}}{d}, 1\right) \times\left(1-e^{\frac{-d}{d_{2}}}\right)+e^{\frac{-d}{d_{2}}},
$$

where $d$ is distance between $\mathrm{Tx}$ and $\mathrm{Rx}, d_{1}$ is a parameter set to 18 meters, and $d_{2}$ to 36 meters. Note that UMi LOS probability model distinguished between LOS and a generic non-LOS state, where LOS blockage is assumed to occur predominantly due to static objects (i.e., akin to NLOSb). For illustration purposes, we use the states generated by the two models to calculate the path loss for a Tx-Rx pair that moves apart at $1 \mathrm{~m} / \mathrm{s}$ starting from 1 to 500 meters. We use the parameters for urban medium density (Figs. 2(b) and 4(b)). For LOS and NLOSb path loss parameters, we use the values based on measurements reported in [5]. For NLOSv, we use the multiple knife-edge attenuation model described in [5]. However, to make the difference between states more clearly visible, we simplify the NLOSv model so that it adds a constant $8 \mathrm{~dB}$ attenuation compared to free-space path loss. Also for clarity reasons, we show path loss only (i.e., without shadow fading). Fig. 7 shows the path loss results for the proposed model and 3GPP UMi LOS probability model [8]. Since UMi model does not model dependency on the previous LOS state, the number of transitions between the states is considerably higher 


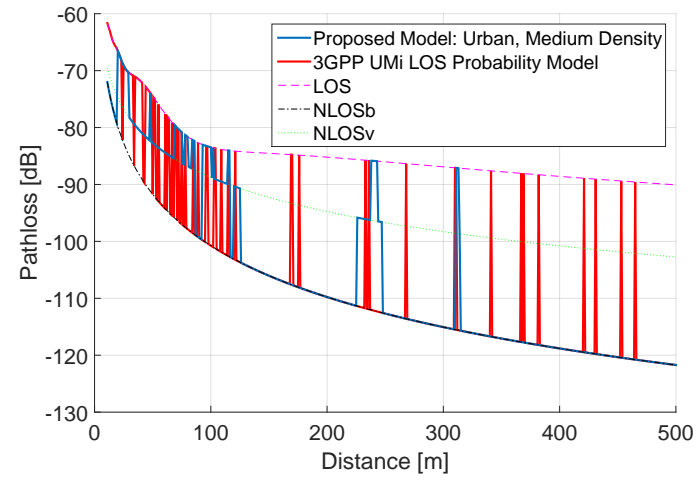

Figure 7. Comparison of path loss generated by proposed model and 3GPP UMi LOS probability model for a single V2V link with Tx and Rx moving apart from 0 to $500 \mathrm{~m}$ with relative speed of $1 \mathrm{~m} / \mathrm{s}$. For reference, path loss for LOS, NLOSv, and NLOSb states are plotted.

than in the proposed model, particularly when the probability of LOS is close to $50 \%$ (i.e., between 50 and 100 meters). This is an unrealistic behavior, since two vehicles can not move between LOS and non-LOS states so rapidly. The proposed model, on the other hand, takes into account the previous state and smoothly evolves the link between LOS, NLOSb, and NLOSv states. While Fig. 7 shows a single realization of state changes and resulting path loss, we ran simulations for a large number $\left(10^{5}\right)$ of $\mathrm{V} 2 \mathrm{~V}$ pairs with distances between 0 and $500 \mathrm{~m}$. UMi model resulted in an average state change every 5 seconds, while the proposed model averaged one state change every 17 seconds.

The repercussion of a more realistic LOS evolution are manifold. Specifically, performing simulations without the proposed model and using simple probability models such as UMi results in: i) inaccurate estimate of interference, since the calculated interference contributions from specific vehicles will be more sustained than what a simple model estimates; ii) overestimating the benefits of retransmissions; a link in NLOS state is likely to stay in that state for a longer period of time than what is estimated by a simple model, thus making the retransmissions less effective; iii) erroneous estimate of performance for applications requiring continuous transmission between two vehicles, since the link duration will be impacted by the unrealistically high number of transitions between the states.

The result in Fig. 7 also indicates why shadowing correlation models are not sufficient for modeling time- and spaceevolved V2V links. Shadowing decorrelation distance in urban and highway environment for $\mathrm{V} 2 \mathrm{~V}$ links is on the order of tens of meters (e.g., Abbas et al. [16] report decorrelation distances of $5 \mathrm{~m}$ in urban and $30 \mathrm{~m}$ in highway environment) and is assumed do be independent of the Tx-Rx distance. However, Fig. 7] shows that a single decorrelation distance value cannot capture the changing behavior as Tx-Rx distance changes: at low distances, the LOS decorrelation distance is high and decreases with increasing Tx-Rx distance, whereas NLOSb decorrelation distance increases with increasing $\mathrm{Tx}-\mathrm{Rx}$ distance. By using distance-dependent transition probabilities, the employed model is capable of capturing this behavior.

\section{CONCLUSIONS}

We performed a comprehensive analysis of LOS blockage evolution for V2V links in real cities and highways. To efficiently model the time evolution of LOS blockage for V2V links, we employed a three-state Markov chain, which we trained using a large set of realistically simulated V2V links (more than $10^{6}$ $\mathrm{V} 2 \mathrm{~V}$ links in high density scenarios) in urban and highway environments. To enable simple incorporation of LOS blockage evolution in simulations, we performed curve-fitting of the model parameters. The resulting LOS probability and transition probability parameters provide a detailed and realistic evolution of LOS blockage that is a function of Tx-Rx distance, environment (highway/urban), and vehicle density (low/medium/high).

While we focused on $\mathrm{V} 2 \mathrm{~V}$ communication, the methodology we presented can be used for other types of communication (e.g., V2I, D2D with pedestrians carrying the devices, etc.), provided that realistic mobility and map information is available. Future work will include analysis and efficient modeling of crosscorrelation of LOS blockage evolution for spatial consistency of multiple V2V links in geographic proximity.

\section{REFERENCES}

[1] A. Osseiran, F. Boccardi, V. Braun, K. Kusume, P. Marsch, M. Maternia, O. Queseth, M. Schellmann, H. Schotten, H. Taoka et al., "Scenarios for 5G mobile and wireless communications: the vision of the METIS project," IEEE Communications Magazine, vol. 52, no. 5, pp. 26-35, 2014.

[2] ETSI TC ITS, "Intelligent transport systems (ITS); vehicular communications; basic set of applications; part 2: Specification of cooperative awareness basic service," Tech. Rep. EN 302 637-2, 2014.

[3] D. S. Baum, J. Hansen, and J. Salo, "An interim channel model for beyond$3 \mathrm{~g}$ systems: extending the 3gpp spatial channel model (SCM)," in IEEE Vehicular Technology Conference, VTC-Spring, vol. 5, 2005, pp. 3132-3136.

[4] J. Maurer, T. Fugen, T. Schafer, and W. Wiesbeck, "A new inter-vehicle communications (IVC) channel model," in IEEE Vehicular Technology Conference, VTC-Fall, vol. 1, 2004, pp. 9-13.

[5] M. Boban, J. Barros, and O. Tonguz, "Geometry-based vehicle-to-vehicle channel modeling for large-scale simulation," IEEE Transactions on Vehicular Technology, vol. PP, no. 99, pp. 1-1, 2014.

[6] W. Viriyasitavat, M. Boban, H.-M. Tsai, and A. Vasilakos, "Vehicular communications: Survey and challenges of channel and propagation models," IEEE Vehicular Technology Magazine, vol. 10, no. 2, pp. 55-66, 2015.

[7] T. Abbas, J. Kåredal, and F. Tufvesson, "Shadow fading model for vehicleto-vehicle network simulators," in COST IC1004 5th Management Committee and Scientific Meeting, 2012.

[8] "Report ITU-R M.2135-1: Guidelines for evaluation of radio interface technologies for IMT-Advanced," 2009.

[9] Aalto University, BUPT, CMCC, Ericsson, Huawei, Intel, KT Corporation, Nokia, NTT DOCOMO, New York University, Qualcomm, Samsung, University of Bristol, and University of Southern California, "5G channel model for bands up to $100 \mathrm{GHz}$, v2.0," in IEEE Globecom 2015 Workshops, Mar. 2016. [Online]. Available: http://www.5gworkshops.com

[10] H. S. Wang and N. Moayeri, "Finite-state markov channel-a useful model for radio communication channels," Vehicular Technology, IEEE Transactions on, vol. 44, no. 1, pp. 163-171, 1995.

[11] J. pierre Ebert, A. Willig, and A. Wolisz, "A gilbert-elliot bit error model and the efficient use in packet level simulation," TU Berlin, Tech. Rep., 1999.

[12] D. Dhoutaut, A. Regis, and F. Spies, "Impact of radio propagation models in vehicular ad hoc networks simulations," VANET 06: Proceedings of the 3rd international workshop on Vehicular ad hoc networks, pp. 69-78, 2006.

[13] X. Wang, E. Anderson, P. Steenkiste, and F. Bai, "Simulating spatial cross-correlation in vehicular networks," in IEEE Vehicular Networking Conference (VNC). IEEE, 2014, pp. 207-214.

[14] R. Meireles, M. Boban, P. Steenkiste, O. Tonguz, and J. Barros, "Experimental study on the impact of vehicular obstructions in VANETs," in IEEE Vehicular Networking Conference (VNC), Dec. 2010, pp. $338-345$

[15] M. Boban, R. Meireles, J. Barros, P. Steenkiste, and O. K. Tonguz, "TVR tall vehicle relaying in vehicular networks," IEEE Transactions on Mobile Computing, vol. 13, no. 5, pp. 1118-1131, May 2014.

[16] T. Abbas, K. Sjöberg, J. Karedal, and F. Tufvesson, "A measurement based shadow fading model for vehicle-to-vehicle network simulations," International Journal of Antennas and Propagation, vol. 2015, 2015.

[17] M. Behrisch, L. Bieker, J. Erdmann, and D. Krajzewicz, "SUMO-simulation of urban mobility," in The Third International Conference on Advances in System Simulation (SIMUL), Barcelona, Spain, 2011.

[18] R. W. Rothery, "Car following models," In Trac Flow Theory, 1992.

[19] M. Ferreira, H. Conceição, R. Fernandes, and O. K. Tonguz, "Stereoscopic Aerial Photography: An Alternative to Model-Based Urban Mobility Approaches," in Proceedings of the Sixth ACM International Workshop on VehiculAr Inter-NETworking (VANET). ACM New York, NY, USA, 2009.

[20] N. Wisitpongphan, F. Bai, P. Mudalige, V. Sadekar, and O. K. Tonguz, "Routing in sparse vehicular ad hoc wireless networks," IEEE Journal on Selected Areas in Communications, vol. 25, no. 8, pp. 1538-1556, Oct. 2007.

[21] M. Haklay and P. Weber, "Openstreetmap: User-generated street maps," IEEE Pervasive Computing, vol. 7, no. 4, pp. 12-18, 2008. 